\title{
MATCHING MODES BETWEEN HIRFL AND CSR*
}

\author{
J.Y. Tang, H.H. Li, Y.J. Yuan \\ Institute of Modern Physics, Lanzhou 730000, China
}

\section{Abstract}

National key scientific project 'HIRFL-CSR Cooler Storage Ring' makes use of existing HIRFL as its pre-accelerator. In order to take the full capability of HIRFL, we have studied in detailed the matching modes between HIRFL and CSR. It is proposed to use two matching modes: direct matching between SFC (HIRFL injector cyclotron) and CSRm (CSR main ring), three-cascade matching of SFC, SSC (HIRFL main cyclotron) and CSRm. With these combinations, better beam transmission efficiency, better beam utilization efficiency of HIRFL-CSR accelerator complex and better operation efficiency of HIRFL can be obtained. In the first case, SSC can be used simultaneously in other purposes, either to accelerate medium energy heavy ions or to accelerate protons combined with another small cyclotron.

\section{INTRODUCTION}

HIRFL accelerator facility consists of two isochronous cyclotrons, the injector cyclotron SFC $(K=69)$ and the main cyclotron SSC $(\mathrm{K}=450)$. Now an extending project CSR Cooler Storage Rings (main ring CSRm and experimental ring CSRe) is under construction [1]. The main ring CSRm uses the two cyclotrons as the upstream accelerators. The main parameters of SFC, SSC and CSRm are listed in table 1. In order to get optimal beam transmission efficiency, beam utilization and accelerator utilization, the combination of the accelerators, the possible matching modes have been studied according to the different ion species and energy range. The basic considerations are to inject the SFC beam into CSRm directly to the greatest extent and to use SFC and SSC together for getting higher energy beams from CSRm in the case of medium and heavy ions.

\section{DIRECT INJECTION TO CSRM FROM SFC}

The maximum beam magnetic rigidity is $1.2 \mathrm{Tm}$, and the minimum beam magnetic rigidity can be lowered to $0.76 \mathrm{Tm}$. So, the extracted beam from SFC working within $1.1 \sim 1.2 \mathrm{Tm}$ is quite suitable to be sent to CSRm, either with stripping for light ions or without stripping for medium and heavy ions. The stripper decreases largely the beam current in the case of medium and heavy ions. In doing so, the total transfer efficiency from the ion source to CSRm can be increased as it passes the low

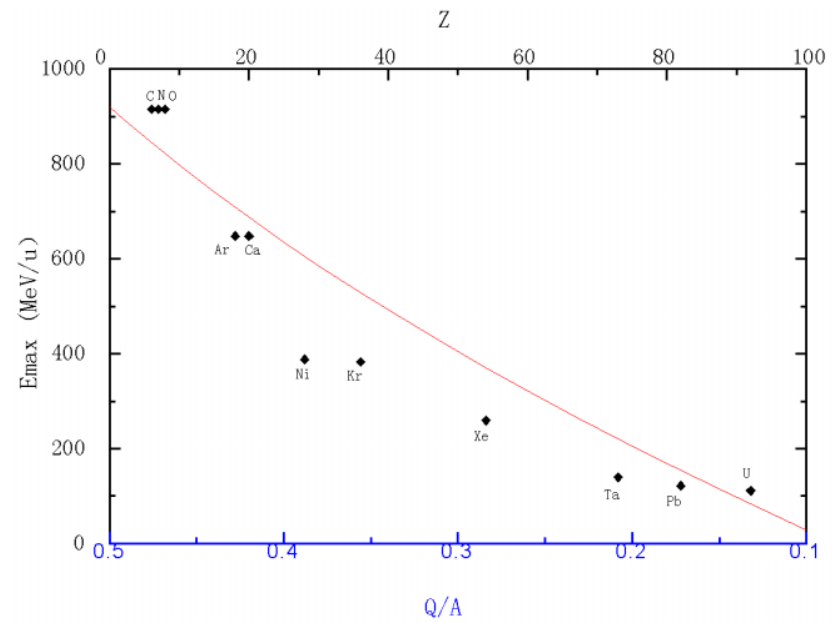

Figure 1. Maximum energy from direct SFC-CSRm matching

Solid line for Emax- $Q / A$ value lower abscissa Diamond symbols for Emax-Z upper abscissa

Table 1 Main parameters for SFC, SSC and CSRm

\begin{tabular}{|c|c|c|c|c|}
\hline & \multirow[t]{2}{*}{ SFC } & \multirow[t]{2}{*}{ SSC } & \multicolumn{2}{|c|}{ CSRm } \\
\hline & & & Accel. & Accum. \\
\hline Energy constant & 69 & 450 & & \\
\hline Max. B field (T) & 1.6 (mean) & 1.6 (gap) & \multicolumn{2}{|c|}{1.4 (dipole) } \\
\hline $\mathrm{RF}$ frequency $(\mathrm{MHz})$ & $5.5-16.5$ & $6.5-14$ & $0.25-1.7$ & $6.0-14.0$ \\
\hline $\mathrm{RF}$ harmonics & 1,3 & $2,4,6$ & 1 & 16,32 \\
\hline Injection radius (m) & $0.025,0.03$ & 1.0 & & \\
\hline Extraction radius (m) & 0.75 & 3.207 & & \\
\hline Circumference (m) & 4.71 (ext.) & 20.15 (ext.) & & .00 \\
\hline Min. mag. rigidity (Tm) & 0.6 (ext.) & 1.5 (ext.) & & 76 \\
\hline Max. mag. rigidity $(\mathrm{Tm})$ & 1.2 (ext.) & $3.05(\mathrm{ext})$ & & 64 \\
\hline
\end{tabular}

\footnotetext{
${ }^{*}$ Supported by HIRFL-CSR cooler storage rings project
} 
efficient SSC. In the mean time, SSC can be used either to accelerate the SFC beam to higher energy or to accelerate proton beam from another injector (PDC) for the experiments in the present experiment stations in the share-time mode with CSRm injection time [2]. Because the maximum injection magnetic rigidity is $0.935 \mathrm{Tm}$ and the stripper is common for the beam either to CSRm or to SSC, so SFC can accelerate ions within 0.76 0.935Tm range (after stripping or with stripper offline) when the beam is delivered to CSRm as well to SSC with the share-time mode. Another limitation from CSRm is the minimum beam energy requirement of $7.3 \mathrm{MeV} / \mathrm{u}$ due to the minimum high voltage $4 \mathrm{kV}$ of the electron cooler, which may be lowered in future. Without considering this factor, the maximum available beam energies from CSRm are shown in Figure 1, for all kinds of elements. If the highest beam energy from CSRm is required in the case of medium and heavy ions, the three acceleration stages of SFC-SSC-CSRm should be used.

When using the direct matching between SFC and CSRm, the injection method to CSRm may be multiple multi-turns plus electron cooling in which the matching between the RF systems of the two accelerators can be ignored, and may be multi-turn plus RF stacking plus electron cooling in which the accumulating RF frequency should be the multiple of SFC RF. For the latter case in which the highest injection efficiency can be obtained, it is just possible by slightly changing the SFC extraction radius $\left(R_{\text {ext }}=0.754 \mathrm{~m}\right.$ instead of $\left.0.750 \mathrm{~m}\right)$ to get the circumference ratio of 34 between SFC and CSRm. So, the accumulating RF cavity can adopt the harmonics of 17, 34 and 68, as the SFC cyclotron uses a subharmonic buncher [1] (see table 2).

\section{MATCHING OF SFC-SSC-CSRM}

In order to get higher energy beam of medium and heavy energy from CSRm, it is needed to inject the beams with higher Q/A value. Therefore, the stripping after further acceleration in SSC is required. Two stages of stripping (after SFC and SSC) are applicable, but the gain in $\mathrm{Q} / \mathrm{A}$ value is very limited and big beam loss at the first stripper is very important [3]. So is usually only the stripper after SSC set online.

For the existing HIRFL, there are four matching modes of SFC and SSC harmonics $\left(h_{S F C}=1,3\right.$ and $\left.h_{S S C}=2,4,6\right)$ [4]. Considering that it is not necessary to inject the light ions with the energy higher than $27 \mathrm{MeV} / \mathrm{u}$, only two modes are considered here: 1$) h_{S F C}=3$ and $h_{S S C}=4$ for light and medium ions; 2) $h_{S F C}=3$ and $h_{S S C}=6$ for heavy ions. The two modes are overlapped in energy range, but the second one is favorable due to the higher energy gain per turn. The reason for excluding the energy range bigger than $27 \mathrm{MeV} / \mathrm{u}$ is that the cooling time will increase largely with high energy.

As in the case of direct injection from SFC, the injection to CSRm from SSC has two methods [5,6]: one is multiple multi-turns plus electron cooling in which the matching between the RF systems of the two accelerators can be ignored, and the other is multi-turn plus RF stacking plus electron cooling. For the latter case, the CSRm circumference has been chosen to be 8 times of SSC's at extraction, and the RF frequency range same as SSC's. The RF harmonic numbers are $h_{C S R m}=32$ and 48 in the two cases, respectively. The main parameters of the three accelerators are shown in table 3. Figure 2 and 3 show the maximum available beam energy and the

Table 2, SFC and CSRm acceleration range with RF stacking method

\begin{tabular}{ccccccc}
\hline$h_{S F C}$ & $\mathbf{h}_{\text {CSRm }}$ & $\mathbf{Q} / \mathbf{A}$ & $\begin{array}{c}f_{S F C} \\
/ \mathrm{MHz}\end{array}$ & $\begin{array}{c}E_{S F C} \\
/\left(\mathrm{MeV} \cdot \mathrm{u}^{-1}\right)\end{array}$ & $f_{C S R m} / \mathrm{MHz}$ & $\begin{array}{c}E_{C S R m} \\
/\left(\mathrm{MeV} \cdot \mathrm{u}^{-1}\right)\end{array}$ \\
\hline 1 & 34 & $0.255-0.5$ & $6.0-11.773$ & $4.18-16.37$ & $6.0-11.773$ & $305.1-915.5$ \\
1 & 68 & $0.234-0.255$ & $5.5-6.0$ & $3.50-4.18$ & $11.0-12.0$ & $262.2-305.1$ \\
3 & 17 & $0.198-0.234$ & $14.0-16.5$ & $2.52-3.50$ & $7.0-8.25$ & $193.9-262.2$ \\
3 & 34 & $0.085-0.198$ & $6.0-14.0$ & $0.46-2.52$ & $6.0-14.0$ & $38.7-193.9$ \\
3 & 68 & $0.078-0.085$ & $5.5-6.0$ & $0.39-0.46$ & $11.0-12.0$ & $32.6-38.7$ \\
\hline
\end{tabular}

Note $h_{S F C}$ for SFC acceleration harmonic number $h_{C S R m}$ for CSRm accumulation $f_{S F C}$ for SFC RF frequency, $E_{S F C}$ and $E_{C S R m}$ for the extraction energies from SFC and CSRm. The SFC magnetic rigidity used for the calculation is $1.15 \mathrm{Tm}$.

Table 3, matching of the parameters for SFC-SSC-CSRm acceleration

\begin{tabular}{|c|c|c|c|c|c|c|}
\hline Mode & $\begin{array}{c}h \\
S F C / S S C / C S R m\end{array}$ & $\begin{array}{c}f_{S F C} \\
/ \mathrm{MHz}\end{array}$ & $\begin{array}{c}f_{S S C} \\
/ \mathrm{MHz}\end{array}$ & $\begin{array}{l}f_{C S R m} \\
/ \mathrm{MHz}\end{array}$ & $\begin{array}{c}E_{S F C} \\
/\left(\mathrm{MeV} \cdot \mathrm{u}^{-1}\right)\end{array}$ & $\begin{array}{c}E_{S S C} \\
/\left(\mathrm{MeV} \cdot \mathrm{u}^{-1}\right)\end{array}$ \\
\hline 1 & $3 / 4 / 32$ & $6.50 \quad 14.00$ & $6.50 \quad 14.00$ & $6.50 \quad 14.00$ & $0.54 \quad 2.52$ & $5.61 \quad 26.9$ \\
\hline 2 & $3 / 6 / 48$ & $5.50 \quad 9.33$ & $8.25 \quad 14.00$ & $8.25 \quad 9.33$ & $0.39 \quad 1.12$ & $4.00 \quad 11.67$ \\
\hline
\end{tabular}


acceleration Q-A range. The dashed line represents the limitation from the nowaday level of ECR ion source [7].

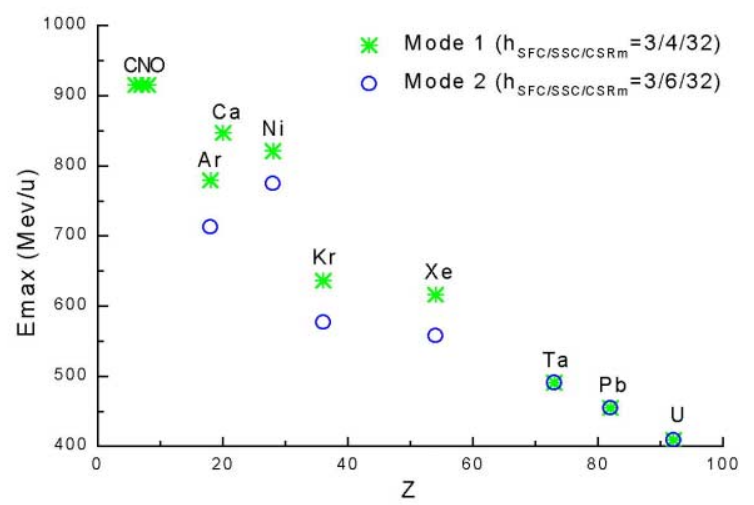

Figure 2. Maximum energy from SFC-SSC-CSRm matching

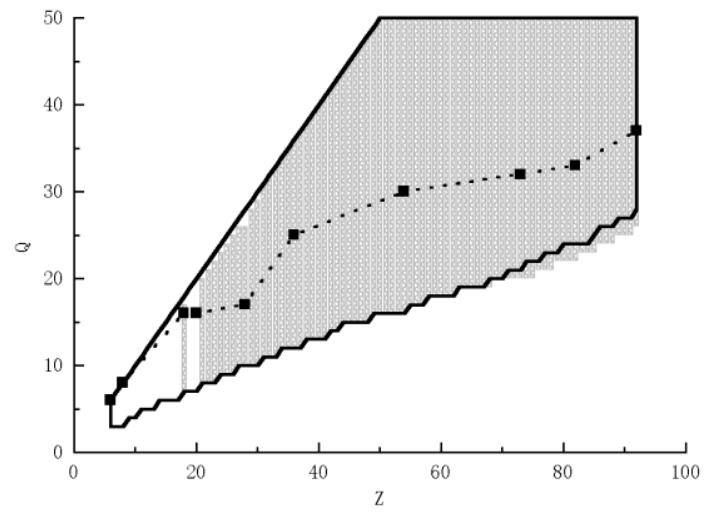

Figure 3 Charge State $Q$ range for SFC-SSC-CSRm combination

Solid line enclosed area for mode $1\left(h_{S F C / S S C / C S R m}=3 / 4 / 32\right)$

Shadow area for mode $2\left(h_{S F C / S S C / C S R m}=3 / 6 / 32\right)$

Dashed line for the ECR ion source limitation

\section{CONCLUSIONS}

HIRFL-CSR accelerator complex can work in the different combination modes, in order to get the best beam transmission efficiency and the best utilization efficiency of the accelerators.

1) For light ions (Carbon-Argon), it is desirable to use the direct matching of SFC-CSRm, as the available $\mathrm{Q} / \mathrm{A}$ values from ECR ion source are high. In doing so, the spare cyclotron SSC can be used to accelerate either medium energy heavy ions from SFC or proton beam from PDC.

2) For medium ions (Argon-Xenon) and heavy ions (Xenon-Uranium), the matching of SFC-SSC-CSRm should be the good choice as it provides large energy range from CSRm. The matching of SFC-CSRm is possible when the high voltage of the electron cooler can be decreased below $4 \mathrm{kV}$.

3) Whether SFC-CSRm combination or SFC-SSC-CSRm combination is used, CSRm can use any of the two accumulation method: multiple multi-turns plus electron cooling and multi-turn plus RF stacking plus electron cooling.

\section{REFERENCES}

[1] Preliminary Design of HIRFL CSR Project, Beijing Academe of Construction Design and Research, 1999.4

[2] Tang J Y, Yin Q M et al., Scheme of Accelerating Protons at HIRFL, Proceedings of Cyclic Accelerators and Their Applications, 2000. 83-88

[3] Tang J Y, Wang Y F and Yin Q M, Nuclear Physics Review (in Chinese), 1999, 17(2): 95-99

[4] Tang J Y and Yin Q M, High Power Laser and Particle Beams (in Chinese), 1999, $11(6): 765-770$

[5] Yuan Y J et al., Simulation of RF Stacking and Multiple Single-turn Injection and Multi-turn Injection, Proceedings of 14th International Conference on Cyclotron and their Application, Cape Town, South Africa, World Scientific Publishing Co. 1995.10

[6] Yuan Y J et al., Simulation of RF Stacking Combined with Cooling Effects, Proceedings of 5th European Particle Accelerator Conference (EPAC), Sitges, Barcelona, 1996.6

[7] Preliminary Design of Superconducting High Charge-state ECR Ion Source, Institute of Modern Physics, Internal Report, 2000 